\title{
Subject Index to Volume 26
}

Acidemia, propionic, carnitine metabolism in, 63

Adenosine, concentration, cord blood, vaginal delivery and cesarean section, 106

Afterload, left ventricle adaptations to, preterm lamb, 336

Age

intestinal response to hemorrhage and, developing piglet, 102

parathyroid bormone responsiveness and immature rat, 54

plasma levels of $\beta$-casomorphin-7 after milk intake related to, dog, 34

reduction of insulin-like growth factor I by protein restriction related to, rat, 415

Agglutination test, antibody detection, autoimmune neutropenia, infant, 246

Air-blood barrier, development, human fetal lung, 88

Airway, occlusion, genioglossus and diaphragm activity during, infant, 583

Alanine, transport, plasma membrane vesicles, pregnant rat liver, 448

Alkalosis, respiratory, indomethacin effect on cerebral blood flow regulation during, newborn piglet, 593

Alkaptonuria, ascorbic acid effect, children and adults, 140

Allergy, serum immunoglobulin $E$ levels in, role of $F_{c}$ receptors, 49

American Pediatric Society, presidential address 1989,287

Amino acid, plasma concentrations, protein intake and, infant, 614

Aminophylline, effect on diaphragmatic blood flow, piglet, 196

Antibody

binding patterns, immunoblot analysis, Lyme disease, 377

detection, immunofluorescence and agglutination test, infant, 246

group B Streptococcus type-specific, transplacental or enteral transfer, suckling rat, 629

Antigen, absorption, bacterial diarrhea, rabbit, 237

Antigenuria, Haemophilus influenzae type b, in asymptomatic carriers, 491

Antilipolytic hormone, adipocyte response, fat cells, infants and adults, 255

Antioxidant

defense mechanisms, developmental patterns, human erythrocytes, 366

hyperoxia effect, type II cells, neonatal rat, 400

total radical trapping capacity, cord blood plasma, term and preterm neonate, 20

Antioxidant enzyme, maturation, sex differ-

Apnea ences, fetal rabbit lung, 16

at-risk infants, reflex modification procedures, 576

obstructive, genioglossus and diaphragm activity during, infant, 583

Ascorbic acid, effect on benzoquinone acetic acid, alkaptonuria, adults and children, 140

L-Asparaginase, direct long-term effects, cultured pancreatic islets, 158
Asphyxia

cerebral hypoperfusion after, role of oxygen free radicals, newborn lamb, 215

perinatal, adenosine effect, vaginal delivery, 106

Atrial natriuretic peptide, renal and cardiovascular effects, ovine fetus, 1

Bacteria, opsonization, biochemical abnormalities of neonatal C3, 116

Benzoquinone acetic acid, ascorbic acid ef fect, alkaptonuria, adults and children, 140

Bilirubin

detoxification, extracorporeal bilirubin oxidase reactor, rat, 452

neurotoxicity, nuclear magnetic resonance spectroscopic study, rat, 356

Biotinase, deficiency, subacute necrotizing encephalomyelopathy caused by, 260

Birth, afterload alterations, adaptations of left ventricle to, preterm lamb, 336

Blood flow

atrial natriuretic peptide effect, fetal sheep,

cerebral, regulation, indomethacin effect, respiratory alkalosis, newborn piglet, 593

diaphragmatic, aminophylline effect, piglet, 196

during cardiopulmonary resuscitation with simultaneous chest compression and ventilation, infant pig, 558

intestinal, response to hemorrhage, piglet, 102

Borrelia burgdorferi, antibody binding to polypeptides of, immunoblot analysis, Lyme disease, 377

Brain

alternate energy fuels, after bypoxia, neonatal mice, 478

cingulate sulcus, development, preterm in fants, 598

injury

cingulate sulcus maturation and, preterm infants, 598

hypoxic-ischemic, regional cerebral glucose use and, rat, 208

myo-inositol levels, effect of chronic hypernatremia on, mice, 482

Brainstem, altered sensory processing, assessment, reflex modification procedures, 576

Breathing: see Respiration

Bronchopulmonary dysplasia

elastase/proteinase inhibitor imbalance in infants on prolonged ventilation, 351

furosemide effects, body water compartments, infant, 121

Câlcium

transport

intestinal Golgi vesicles, developing rat, 58

placental, fetal control, rat, 109

Carbamyl phosphate synthetase, radiochro matographic measurement, enzymedeficient human liver, 77
Carboxylation, vitamin K-dependent, maturation, fetal and neonatal rat liver, 370

Cardiac output

low, metabolic response, lamb, 180

low dose dopamine effect, after open heart surgery, 200

Cardiopulmonary resuscitation, simultaneous chest compression and ventilation technique, blood flow during, infant pig, 558

Carnitine, metabolism, in propionic acidemia, 63

B-Casomorphin-7, plasma levels, after milk ingestion, age effect, dog, 34

Cerebral artery, blood flow velocity characteristics, maturational differences, neonate, 588

Chemotherapy, effect on tumor burden and energy utilization, newly diagnosed acute lymphoblastic leukemia, 154

Chromatography, affinity, lactoferrin purification by, 618

Cingulate sulcus, development, preterm infants, 598

Collagen, lung, neonatal protein deprivation and, rat, 125

Colon, epidermal growth factor and transforming growth factor immunoreactivity, human fetus, 25

Colony-stimulating factor, recombinant human granulocyte-macrophage, effect on cord blood granulocyte function, 43

Colostrum, removal, recovery, and characterization of immunoglobulin from, 623

Complement, third component, biochemical abnormalities, neonate, 116

Complementation analysis, in peroxisome disorders, 67

Copper, absorption, measurement technique comparison, very low birth weight infants, 298

Cord blood

adenosine concentration, vaginal delivery and cesarean section, 106

granulocyte functions, effects of recombjnant buman granulocyte-macrophage colony stimulating factor, 43

monocytes, herpes simplex replication in, human neonate, 135

plasma radical trapping capacity, term and preterm neonate, 20

Cortisol, renal effects, gestational changes, ovine fetus, 6

Cystine, lysosomal, depletion by mercaptoethylgluconamide, 73

Cystinosis, nephropathic, cystine-depleting action of mercaptoethylgluconamide, 73

Deoxyribonucleic acid, immobilized singlestranded, purification of human lactoferrin, 618

Dexamethasone, prenatal exposure, effect on heart rate control mechanisms, rat, 554

$D-\beta$-Hydroxybutyrate, as alternate energy fuel, posthypoxia brain cells, neonatal mice, 478 
Diaphragm, electromyographic activity, during obstructive apnea and airway occlusion, infant, 583

Diet, protein restriction, age-related reduction of insulin-like growth factor-I, rat, 415

1,25-Dihydroxyvitamin $D_{3}$, production and metabolic clearance rate, pregnant sheep and chronically catheterized fetus, 633

Dopamine, low dose, effect on hemodynamic and renal function, 200

Ductus arteriosus, oxygen-related prostaglandin synthesis in, fetal lamb, 330

Elastase/proteinase inhibitor, imbalance, during prolonged ventilation, infant, 351

Elastin, lung, neonatal protein deprivation and, rat, 125

Electrocardiography, QT prolongation, chick embryo, 11

Encephalomyelopathy, subacute necrotizing, caused by biotinidase deficiency, 260

Energy, expenditure, newly diagnosed acute lymphoblastic leukemia, 154

Energy substrate, total parenteral nutrition, neonate, 290

Enzyme, antioxidant, hyperoxia effect, type II cells, neonatal rat, 400

Epidermal growth factor, immunoreactivity, human fetal gut, 25

Erythrocyte, antioxidant defense mechanisms, developmental patterns, 366

Escherichia coli, diarrhea, antigen absorption in, rabbit, 237

Eucaryotic cells, Haemophilus influenzae type $b$ adherence to, kinetics, 500

European Society for Paediatric Gastroenterology

abstracts of annual meeting, 267

author index to abstracts, 284

European Society for Pediatric Research, abstracts of annual meeting, 504

Exhalation, passive, respiratory system mechanics, preterm lamb, 425

Fatty acid, total, total parenteral nutrition, neonate, 290

Fetus

cardiovascular effects of nifedipine, rat, 442

1,25-dihydroxyvitamin $D_{3}$ production and metabolic clearance rates, primed infusion technique, sheep, 633

fluid homeostasis, role, atrial natriuretic peptide, sheep, 1

beart rate control mechanisms, dexamethasone and terbutaline effect, rat, 554

hematopoietic growth factors, developmental regulation, 531

iron utilization, prolonged intrauterine hyperinsulinemia effect, sheep, 467

lung

air-blood barrier development, 88

antioxidant enzyme maturation, sex differences, rabbit, 16

prostaglandin synthesis, oxygen-related, ductus arteriosus, lamb, 330

renal effects of cortisol, gestational changes, sheep, 6

Sanfilippo syndrome, type D, spectrophotometric detection, 462

Food, thermic effect, energy metabolism and, newly diagnosed acute lymphoblastic leukemia, 154
Free radical, trapping ability, cord blood plasma, term and preterm neonate, 20

Furosemide, effect on body water compartments, bronchopulmonary dysplasia infant, 121

Galanin, growth hormone secretion and, normal and short stature children, 316

Gastric motikity, effece of postnatal age, rabbit, 458

Gastroesophageal reflux, developmental differences of lower esophageal sphincter, cat, 39

Gender, antioxidant enzyme development and, fetal rabbit lung, 16

Genioglossus, electromyographic activity, during obstructive apnea and airway occlusion, infant, 583

Glucose, regional cerebral utilization, immature rat, 208

Glycoprotein, intestinal, synthesis, tissuespecific characteristics, suckling rat colon, 232

Golgi membranes, intestinal, calcium transport by, developing rat, 58

Granulocyte, effect of recombinant human granulocyte-macrophage colony-stimulating factor on, neonate, 395

Growth and development

agonist-mediated gastric smooth muscle contraction, developmental changes, rabbit, 458

air-blood barrier development, human fetal lung, 88

antioxidant defense mechanisms, human erythrocytes, 366

calcium transport by intestinal Golgi vesicles, developing rat, 58

cerebral velocity waveform characteristics. effect of gestational age on, 588

chemotherapy effect, acute lymphoblastic leukemia, 154

cingulate sulcus development, preterm infants, 598

developmental differences in metabolism, during oxygen deficit and recovery, lamb, 180

effect of sodium-deficient diet, rat, 608

galanin effect on growth hormone secretion, normal and short stature, 316

heart rate variation, first 6 months of life, 343

hematopoietic growth factors, developmental regulation, 531

intestinal response to hemorrhage, piglet, 102

lower esophageal sphincter modulation, developmental differences, cat, 39

lung antioxidant enzyme maturation, sex differences, fetal rabbit, 16

lung maturation, ontogeny of surfactant apoproteins, rat, 167

malnutrition effect on insulin-like growth factor I cholestatic rat, 410

mitochondrial antioxidant defense, guinea pig, 220

papillary muscles, electrophysiologic properties, rabbit, 543

plasma $\beta$-casomorphin- 7 after milk ingestion, neonatal and adult dogs, 34

protein intake in early infancy, 614

renal effects of cortisol, gestational changes, ovine fetus, 6

renal response to parathryroid hormone, immature rat, 54 role of growth hormone-releasing hormone in physiologic release of growth hormone, 404

tracheal cartilage mechanics, developmental differences, preterm lamb, 429

upper intestinal epithelial changes, weaning effect, guinea pig, 31

vascular resistance distribution, developmental effects, hypoxia and indomethacin, lamb lung, 325

vitamin $\mathrm{K}$-dependent carboxylation, maturation, fetal and neonatal rat liver, 370

Growth hormone

nocturnal release, normal children, 404

physiologic release, role of growth hormone-releasing hormone, 404

secretion, galanin effect, normal and short stature children, 316

serum concentration, nocturnal, testosterone infusion effect, pubertal boys, 320

with reduced protein diet, uremic rat, 204

Growth hormone-releasing hormone, immunoreactive, nocturnal release, normal children, 404

Growth retardation, chronic renal failure with, insulin-like growth factor binding protein levels, 308

Haemophilus influenzae type b

adherence to eucaryotic cells, kinetics of 500

meningitis, low-dose polymyxin B treatment, infant rat, 496

polysaccharide antigen, urinary excretion, nasopharyngeal carriers. 491

Heart, mitochondrial antioxidant defense, perinatal development, guinea pig, 220

Heart rate

atrial natriuretic peptide effect, fetai sheep,

control mechanisms, effects of prenatal dexamethasone and terbutaline, rat, 554

variability, spectral analysis, full-term infants, 188

variation, development, first 6 months of life, 343

Heart ventricle, contractility and performance, during pressure loading, preterm lamb, 336

Hematopoiesis, developmental regulation of growth factors, fetus and neonate, 531

Heme oxygenase, inhibition by metalloporphyrins, adult rat intestine, 362

Hemorrhage, intestinal response, age-related differences, piglet, 102

Herpes simplex, replication, blood monocytes and placental macrophages, human neonate, 135

Homovanillic acid, urinary levels, neuroblastoma screening program, Japan, 603

Howland Award Acceptance, 390

Howland Award Presentation, 388

Hyaline membrane disease

respiratory mechanics, during passive exhalation, preterm lamb, 425

surfactant therapy, treatment of patent ductus arteriosus after, baboon, 565

Hyperinsulinemia, intrauterine, effect on iron utilization, fetal sheep, 467

Hyperketonemia, cardiac function during, spectroscopic study, dog, 536

Hypernatremia, chronic, effect on brain myo-inositol levels, mice, 482 